logos_i_ethos_2_(33)_2012, s.7-24

Вячеслав Моисеев

\title{
Наука и религия: два образа веры
}

\section{Введение}

В современной европейской культуре бытует мнение о несоизмеримости науки и религии, о пропасти, разделяющей веру и знание. На фоне такого рода установки задача поиска некоторых оснований единства науки и религии является как актуаль-

Моисеев Вячеслав Иванович - доктор философских наук, профессор, заведующий кафедрой философии Московского государственного медико-стоматологического университета, профессор Института философии РАН. ной, так и представляющейся весьма труднодостижимой. Одним из основных препятствий к решению этой задачи является явное или неявное убеждение большинства в принципиальной разнице процедур обоснования научного и религиозного опыта. Если истины веры исходят от самого Бога и потому представляют из себя нечто незыблемое, окончательное и сверхчеловеческое, то истины научного знания - это результат побед или поражений конечного и ограниченного человеческого разума, и потому они никогда не носят окончательного характера, постоянно уточняются и пересматриваются. Сфера религиозного опыта зиждется таким образом на некоторой незыблемой скале богооткровенных догматов, сфера науки вечно пребывает в непрерывном сомнении и поиске. Религиозный опыт представляет собой нечто подобное твердому телу в области человеческого бытия, научный опыт - некое жидкое и вечно текучее начало. С этой же идеей связано и представление об Откровении в религии как о некотором первичном событии - Откровении-инсайте, предпосланном всем остальным решениям религиозного сознания.

Самое главное в религиозном опыте дано в начале, все остальное последует этому первичному факту и скорее может только ослабить 
его, чем развить и упрочить. Наоборот, научное знание получает самое ценное в конце процесса познания - как результат всей полноты разного рода научных процедур обоснования, и дальнейшее применение этих процедур позволяет все более усиливать первичные озарения и догадки ученых. В этом смысле поведение религиозного сообщества предстает похожим на поведение хранителей несметных и неземных сокровищ, главная задача которых - сохранить то, что есть, уберечь его от растраты. Поведение же ученых больше напоминает в этой модели деятельность садоводов, самостоятельно создающих свое скромное сокровище - урожай, и получающих его только в итоге своих многочисленных усилий. Такого рода широко распространенную модель отношений науки и религии, знания и веры, я буду называть в этой работе контрастной моделью знания и веры, утверждающей максимальный контраст, несовместимость типов обоснования религиозного и научного опыта. Хотя эта модель имеет свой момент оправдания, но, как я постараюсь показать в дальнейшем, реальный религиозный опыт строится во многом подобно опыту научному, в рамках своего рода аналогической модели отношений науки и религии.

Однако такого рода подход, с другой стороны, должен быть убережен от искуса слишком прямолинейного отождествления религиозного опыта с научным, от своего рода научного редукционизма в понимании феномена религии. Правильнее будет говорить о некоторой единой жизнедеятельности человеческих существ (Жизни, или Культуре, с большой буквы), в которой присутствуют универсальные типы сущностей и деятельностей, по-своему преломляемые в той или иной разновидности человеко-бытия, например, в науке или религии. Одним из таких фундаментальных актов Жизни является акт усиления своего бытия, позволяющий достичь Жизнью более безусловных и абсолютных своих состояний. Наука и Религия представляют из себя, по-видимому, два важнейших проявления этой Воли к Абсолютному. В них нет некоторой врожденной несоизмеримости, и при более глубоком отношении они способны обнаружить один корень, один исток - более подлинное отношение 
к миру. Повсюду так или иначе выражает себя стремление человека к более сильному и подлинному бытию. Во всех формах жизни человек тяготеет к выявлению более сильного бытия и использует для этого разного рода активности. Я буду называть их процедурами обоснования, понимая здесь под обоснованием некоторую универсальную человеческую активность, позволяющую в том или ином смысле повысить степень доверия субъекта к тому или иному событию, состоянию.

\section{$\S 1$ Примеры научных процедур обоснования}

В нашем подходе рассматривается понятие „процедура обоснования”. В общем случае это некоторый акт человеческой деятельности, позволяющий сообщить уверенность и осмысленность тем или иным состояниям человеко-бытия. В определённом смысле процедуры обоснования - это удивительный преобразователь, который в научном познании превращает просто информацию в знание. С этой точки зрения, феномен доказательства и обоснования играет важную роль в определении самого явления знания. Теме обоснования было посвящено множество работ в области философии науки. Здесь можно вспомнить Рассуждение о методе Декарта, Систему логики Джона Милля, развитое учение о дедуктивной выводимости и законах логического следования в математической логике, исследование Л. Витгенштейна О достоверности или работы К. Поппера по философии науки. Среди российских исследователей также можно сослаться на множество работ, например, на исследования 3. А. Сокулер ${ }^{1}$ или Е. П. Никитина ${ }^{2}$. Обширная литература, посвящённая проблемам понимания, представлена в герменевтической традиции. В конечном итоге, вряд ли можно найти автора, который, так или иначе рассматривая феномен знания

3. А. Сокулер, Проблема обоснования знания (Гносеологические кониепции Л. Витгенштейна и К. Поппера), Москва 1988.

2 Е. П. Никитин, Открытие и обоснование, Москва 1988. 
и научной методологии, не касался бы определённым образом проблематики обоснования.

В то же время, как представляется, во всех этих исследованиях не вполне представлен некоторый единый взгляд и интегрирующая перспектива на различные виды процедур обоснования, на то общее, что всех их объединяет и выставляет именно в обсновательном контексте. Если нечто подобное и предполагается, то не вполне структурно. Ниже будет сделана попытка, с одной стороны, обобщить различные виды процедур обоснования, с другой стороны, - предложить более структурные выражения подобной общности. Конечно, автор вполне отдаёт себе отчёт, что проведённое исследование имеет предварительный характер и подразумевает уточнения и изменения в сделанных выводах при более содержательном анализе.

Рассмотрим вначале различные примеры научных процедур обоснования.

Как уже было отмечено, научное познание - это во многом разного рода доказательства и обоснования. Например, в процессе научной деятельности ученый может применять такие процедуры обоснования, как дедукция, индукция, определение понятия, измерение, защита диссертации. Рассмотрим вкратце структуру этих методов обоснования научного знания и научной деятельности.

1. Дедукция. Дедукцией, как известно, называются различные умозаключения, выводы, которые построены в согласии с логическими законами. В общем случае можно утверждать, что дедуктивный вывод имеет вид

$$
\frac{A_{1}, A_{2}, \ldots, A_{n}}{B_{1}, B_{2}, \ldots, B_{m}}
$$

где $A_{1}, A_{2}, \ldots, A_{n}$ - утверждения, являющиеся посылками дедукции, $\mathrm{B}_{1}, \mathrm{~B}_{2}, \ldots, \mathrm{B}_{\mathrm{m}}$ - утверждения, играющие роль заключений дедукции. Сама дедукция есть переход от посылок к заключениям, причем, те и другие должны быть определенной логической формы. 
Для всякого дедуктивного вывода должно выполняться важное свойство - если посылки истинны, то заключения так же должны быть истинными. Это своего рода свойство переноса истинности дедуктивного вывода. Дедуктивные выводы - это как бы трубки, по которым течет истинность, распространяясь от посылок дедукции к заключениям.

2. Индукция. Классическим примером рассуждения по индукции является переход в мысли от частного к общему. В индуктивном выводе мыслитель имеет дело с некоторым классом объектов. Этот класс содержит обычно очень большое число объектов, которые практически невозможно все исследовать. Далее обнаруживается, что некоторое конечное число объектов обладает некоторым свойством Р. На этом основании исследователь может с некоторой вероятностью предполагать, что свойство Р выполняется для всех объектов класса. В отличие от дедукции, индуктивный вывод уже не всегда обладает свойством переноса истинности: даже если посылки индукции истинны, заключение, в лучшем случае, истинно лишь с некоторой вероятностью. Индукция - это как бы „дырявая” трубка, из которой истинность может выливаться.

3. Определение понятия. Индукция и дедукция обосновывают одни суждения на основе других. Но есть обоснования понятий. Одним из наиболее распространенных примеров обоснования понятия является определение понятия. Например, в биологии могут использоваться различные определения жизни: „жизнь есть способ существования белковых тел” или „жизнь есть самоорганизующаяся открытая система”. Множество примеров определения понятий можно найти во всех науках: „сублимация есть процесс использования энергии libido в социально приемлемой форме”, „функция есть однозначное отображение”, „энтропия есть мера неопределенности системы”, и т.д. Отсюда видно, что обычно определение выражается в форме „А есть В”, где А - это определяемое понятие (дефиниендум), В - система определяющих понятий (дефиниенс). Для того чтобы определение было правильным, нужно, в частности, чтобы определяющие понятия уже были понятны к моменту определения 
понятия А. Такая более ранняя понятность может достигаться либо на основе более ранних определений, либо на основе самопонятности понятий, когда они уже настолько очевидны, что не требуют специального определения в рамках некоторой теории. В этом смысле понятия в дефиниенсе В должны быть более первичными (по времени и порядку понимания), чем понятие А. Таким образом, определение понятия также можно было бы изобразить в форме двух уровней:

$\frac{\mathrm{A} \mathrm{есть}\left(\mathrm{B}_{1}, \mathrm{~B}_{2}, \ldots, \mathrm{B}_{\mathrm{m}}\right), \mathrm{B}_{1}, \mathrm{~B}_{2}, \ldots, \mathrm{B}_{\mathrm{m}}}{\mathrm{A}}$

где „А есть $\left(\mathrm{B}_{1}, \mathrm{~B}_{2}, \ldots, \mathrm{B}_{\mathrm{m}}\right)$ ” - некоторая используемая схема определения, $\mathrm{B}_{1}, \mathrm{~B}_{2}, \ldots, \mathrm{B}_{\mathrm{m}}$ - более первичные понятия из дефиниенса, A - определяемое понятие. Само определение предстает в этом случае как перенос понятности (а не истинности) от уже понятных понятий к еще непонятному.

4. Измерение. В науке часто что-то измеряют, например, можно измерить длину, ширину и высоту тела, его вес, объем, можно в психологии измерить степень интеллекта или агрессии, в социологии можно измерить степень совершенства выполнения того или иного действия, и т.д. Любое измерение предполагает измеряемый объект О и шкалу измерения Ш. Шкала обычно представляет из себя множество элементов, которые составляют те или иные степени единицы Е шкалы. Можно использовать следующее сокращенное обозначение для процедуры измерения:

$$
\frac{\mathrm{E}}{\mathrm{O} \mid \alpha \mathrm{E}}
$$

где $\mathrm{O} \mid \alpha \mathrm{E}$ - это измеряемый объект О как степень $\alpha$ единицы Е шкалы. Такая запись выражает процедуру измерения как переход от единицы шкалы к измеряемому объекту, представленному как степень (мера $\alpha$ ) этой единицы. В измерении переносится уже 
не истинность и не понятность, но - измеренность. После проведения измерения объект оказывается измеренным („сосчитанным”) - представленным тем или иным элементом шкалы, степенью единицы шкалы.

\section{$\S 2$. Общая структура научных процедур обоснования}

Во всех приведенных выше примерах мы можем наблюдать некоторую общую структуру обоснования. Везде, во-первых, есть то, что обосновывается. Это

1. дедуктивное заключение в дедукции;

2. индуктивное заключение в индукции;

3. определяемое понятие (дефиниендум) в определении;

4. измеряемый объект в измерении.

Далее будем называть обосновываемое репрезентатом.

Во-вторых, в любой процедуре обоснования мы находим некоторые основания, выведением из которых или подведением под которые осуществляется обоснование репрезентата. Это

1. дедуктивные посылки в дедукции;

2. индуктивные посылки в индукции;

3. определяющие понятия (дефиниенс) в определении;

4. единица шкалы в измерении.

Наконец, можно говорить о самой процедуре обоснования как о некотором специфическом переходе от оснований к репрезентату. Такой переход можно называть актом обоснования. Это

вывод заключений из посылок в дедукции;

вывод заключений из посылок в индукции;

представление дефиниендума через дефиниенс в определении;

процедура соотнесения измеряемого объекта с элементом шкалы в измерении. 
В целом любую научную процедуру обоснования также можно было бы изобразить как двухуровневую структуру

$$
\frac{A_{1}, A_{2}, \ldots, A_{n}}{B_{1}, B_{2}, \ldots, B_{m}}
$$

где $\mathrm{A}_{1}, \mathrm{~A}_{2}, \ldots, \mathrm{A}_{\mathrm{n}}$ - основания, $\mathrm{B}_{1}, \mathrm{~B}_{2}, \ldots, \mathrm{B}_{\mathrm{m}}$ - репрезентаты, и в качестве акта обоснования выступает тот или иной вид перехода от оснований к репрезентатам.

Следует также заметить, что до проведения процедуры обоснования основания и репрезентаты находятся в двух разных состояниях. Если основания считаются чем-то несомненным, очевидным, необходимым (по крайней мере в рамках данной процедуры обоснования), то репрезентаты, наоборот, потому и подвергаются обоснованию, что они изначально рассматриваются как проблематичные, неочевидные, только лишь возможные. Первое состояние - состояние обобщенной необходимости - я буду далее называть L-cmaтусом (от англ. Law - закон), а второе состояние - M-статусом (от англ. May - возможно). Более конкретно, нахождение в L-статусе некоторого состояния X могло бы означать, что оно в том или ином смысле безусловно и максимально, например, оно необходимо есть, исчерпывая собою некоторое пространство возможностей. Наоборот, нахождение в М-статусе дано тогда, когда состояние Х есть лишь нечто условное и немаксимальное, например, - одна из альтернатив, одна из возможностей в некотором более обширном пространстве возможностей.

Приведем примеры L-статусов:

1. B случае дедукции в L-статусе находятся посылки дедуктивного вывода. Например, это могут быть аксиомы или уже доказанные теоремы в некоторой теории. Аксиомы не требуют своего доказательства, они изначально истинны. Следовательно, L-статус здесь - это либо доказанность, либо истинность. Максимальность выражена в этом случае в равенстве степени истинности единице - максимальной мере истинности. 
2. В индуктивном выводе ситуация та же: L-статус посылок индукции выражается в их истинности или доказанности.

3. В определении в L-статусе находятся более первичные понятия из дефиниенса. Здесь L-статус выражается в понятности этих понятий. B пределе такой L-статус обнаружит себя как „понятность из себя" - вспомним определение субстанции у Спинозы как, в частности, „определяемой через себя”. Наоборот, модусы „определяются через другое”, выражая этим более М-статуарное состояние понятности.

4. В измерении единица шкалы обладает L-статусом - она в максимальной степени измерена относительно самой себя, т.е. $\operatorname{deg}(\mathrm{E}, \mathrm{E})=1$, обнаруживая полное соответствие с собой. Другие элементы шкалы в меньшей мере обнаруживают такое соответствие, но в любом случае и они оказываются со-измеримыми единице шкалы. Такая со-измеримость (измеренность) и выражает идею L-статуса в данном случае.

Если до проведения процедур обоснования репрезентаты даны в М-статусе, то после проведения обоснования они должны также перейти в L-статус - в этом, по-видимому, и состоит смысл осуществления различных процедур доказательства и обоснования. Можно сказать и так, что в процедурах обоснования должен переноситься L-cmamyc, в частности, акты обоснования должны обладать этим свойством: они должны переносить L-статус с оснований на репрезентаты. Если нахождение состояния X в L-статусе обозначить как $\mathrm{X} \downarrow_{\mathrm{L}}$, то структуру процедур обоснования теперь более точно можно было бы представить в следующем виде:

$$
\frac{\mathrm{A}_{1} \downarrow_{\mathrm{L}}, \mathrm{A}_{2} \downarrow_{\mathrm{L}}, \ldots, \mathrm{A}_{\mathrm{n}} \downarrow_{\mathrm{L}}}{\mathrm{B}_{1} \downarrow_{\mathrm{L}}, \mathrm{B}_{2} \downarrow_{\mathrm{L}}, \ldots, \mathrm{B}_{\mathrm{m}} \downarrow_{\mathrm{L}}}
$$

Происходит переход не просто от оснований к репрезентатам, но - от оснований в L-статусе к репрезентатам в L-статусе. Процедуры обоснования - это трубки, по которым течет L-статус, pacпространяясь с оснований на репрезентаты. 
Например:

1. дедукция должна переносить истинность с посылок на заключения

2. индукция должна переносить по крайней мере степень истинности с посылок на заключения

3. определение должно переносить понятность с дефиниенса на дефиниендум

4. измерение должно переносить степень со-измерения объекта единице шкалы

Постоянно используя различные процедуры обоснования, научное мышление распространяет разные виды L-статуса (истинность, доказанность, понятность, измеренность, научность и т.д.) с оснований на репрезентаты, с одних - более ранних - репрезентатов на другие. Так энергия L-статуса, как своего рода сила кристаллизации и догматизации мысли, распространяется на все большие пространства смыслов и состояний, пытаясь превратить бытие в нерушимый научный кристалл.

\section{§3. От научных к религиозным процедурам обоснования}

В отличие от контрастной модели отношения религии и науки, реальный опыт религиозной жизни повсеместно обнаруживает примеры использования разного рода процедур обоснования, если и выходящих за рамки только научных обоснований, то вполне выражающих общую структуру идеологии обоснования. Даже в случае мистического инсайта христианская церковь предполагает возможность существования демонической одержимости, в связи с чем требуются дополнительные процедуры обоснования, позволяющие различить формы и степени мистического опыта. То же можно сказать и об авторитете Священного Писания. Этот авторитет в свою очередь стал результатом множества предварительных процедур обоснования в апостольские времена и период раннего христианства, только пройдя через которые, определенный корпус 
текстов получил свою канонизацию. Даже принятие Христа апостолами, как мы знаем из Евангелия, далеко не всегда совершалось столь однозначно и мгновенно, но также имело свою историю испытаний и проверок (апостолы Иоанн и Фома являют собою лишь два крайних полюса этого спектра). Хотя Бог может сообщать человеку истину, но последняя еще должна быть принята человеком как таковая, и здесь даже Бог уважает человеческую свободу. Более того, обоснование как жажда подлинного бытия вполне выражает богочеловеческий замысел человеческого существа.

\section{§4. Примеры религиозных процедур обоснования}

Ниже я попытаюсь привести примеры религиозных процедур обсонования, проделав их первоначальный анализ.

1. Обоснование авторитетом. В этом виде обоснования, столь распространенном среди людей вообще и среди верующих особенно, предполагается существование двух субъектов $\mathrm{S}$ и $\mathrm{S}^{\star}$, один из которых, например, $S$ является, по мнению $S^{\star}$, компетентным в некоторой области О. Кроме того, субъект $\mathrm{S}^{*}$ верит, что $\mathrm{S}$ сообщает правду об этой области. B итоге $\mathrm{S}$ приобретает уверенность, что он также знает область О. Структуру этого обоснования можно было бы изобразить в следующем виде:

\begin{tabular}{l}
$\mathrm{S}$ знает область $\mathrm{O}$ \\
$\mathrm{S}$ сообщает правду $\mathrm{S}^{\star}$ об области $\mathrm{O}$ \\
\hline $\mathrm{S}^{\star}$ знает область $\mathrm{O}$
\end{tabular}

B такого рода процедуре обоснования в качестве L-статуса выступает состояние знания, которое можно было бы назвать S-авторитетной истинностью. Например, посылка „S знает область O" обычно принимается субъектом $\mathrm{S}^{*}$ не просто как научно истинная, но как истинная в мире безусловного доверия к субъекту S. Здесь истинно все то и только то, что освящено утверждениями субъекта S. Только при этих условиях процедура обоснования 
от авторитета начнет переносить L-статус как S-авторитетную истинность. Хочу заметить, что современная наука так же повсеместно использует обоснование авторитетом, поскольку сегодня науку делают огромные сообщества, где уже невозможно все научные знания проверить лично.

2. Согласие с текстом Священного Писания. По-видимому, этот вид обоснования можно представить как некоторый случай обобщенного измерения, при котором эталоном выступает канонический текст $\left(\mathrm{T}_{\mathrm{E}}\right)$, измеряемым объектом, например, тот или иной оцениваемый текст (Т). Обоснование выглядит здесь как обнаружение достаточно высокой степени соответствия оцениваемого текста эталонному. Итак, имеем:

$$
\frac{\mathrm{T}_{\mathrm{E}}}{\mathrm{T} \mid \alpha \mathrm{T}_{\mathrm{E}}}
$$

где $\mathrm{T} \mid \alpha \mathrm{T}_{\mathrm{E}}$ - текст T как степень $\alpha$ соответствия тексту $\mathrm{T}_{\mathrm{E}}$. Степень $\alpha$ является степенью соответствующего L-статуса, который можно было бы назвать, например, священнописанностью. Священнописанность может включать в себя как общегерменевтические составляющие, выясняемые при сравнении любых текстов (и в этой степени в религии применимы общие методы герменевтики), так и некоторые специфические религиозно-герменевтические процедуры сравнения текстов.

3. Принятие Вселенским Собором. Одна из сильнейших и комплексных процедур обоснования в христианстве. Также может быть рассмотрена как разновидность обобщенной процедуры измерения. Обозначим через ВС некоторый эталон религиозности, присущий Вселенскому Собору. Пусть X - оцениваемое Собором явление (текст, учение, человек и т.д.). Тогда процедура обоснования в этом случае примет вид:

$$
\frac{\mathrm{BC}}{\mathrm{X} \mid \alpha \mathrm{BC}}
$$


Можно предположить, что здесь переносится такой L-статус, как вселенская соборность. Этот L-статус соединяет в себе многие более частные религиозные L-статусы - авторитетную истинность, священнописанность и т.д.

Хочу отметить, что разного рода процедуры обоснования типа измерения широко распространены вообще в жизни субъектов, поскольку через такого рода обоснования можно представить различные оценки - как степени соответствия тем или иным субъектным эталонам. Я буду называть такие процедуры обоснования сравнительными обоснованиями. Их общий вид может быть выражен таким образом:

$$
\frac{\mathrm{E}}{\mathrm{X} \mid \alpha \mathrm{E}}
$$

Здесь Е - некоторый субъектный эталон (ценность, идеал, норма и т.д.), $\mathrm{X}$ - оцениваемое явление, $\mathrm{X} \mid \alpha \mathrm{E}$ - X как степень соответствия $\alpha$ эталону. В этом случае переносится L-статус, который может быть назван Е-товостью, т.е. обоснование выражает себя как обнаружение Е-товости репрезентата, возможно, достаточно высокой его степени.

Еще примеры религиозных сравнительных процедур обоснования.

4. Утверждение статуса „отец Церкви”. Как пишет иерей Олег Давыденков в Догматическом богословии, „Формально можно выделить три принципа, по которым тот или иной богослов считается отцом Церкви: 1) Sanctitas vitae - святость жизни; 2) Sanitas doctrinae - здравость учения, истинность, верность учения; 3) Ecclesial declaratio - свидетельство Церкви"'. Здесь мы имеем дело с распадением общей процедуры обоснования на три более частные процедуры. В целом можно использовать такое сокращенное представление этих процедур обоснования: 


$\frac{S V}{X \mid \alpha S V} \frac{S D}{X \mid \beta S D} \frac{E D}{X \mid \gamma E D}$
$X \mid \delta$ Pater

Здесь SV - эталон религиозной святости жизни, SD - эталон здравости учения, ED - эталон Церковного свидетельства, X оцениваемый богослов, Pater - эталон отца Церкви. Итоговое coответствие эталону отца Церкви $(\delta)$ формируется здесь на основе вкладов соответствий ( $\alpha, \beta$ и $\gamma)$ каждому более частному эталону. Соответствующий L-статус в итоговой процедуре обоснования можно было бы назвать отцецерковностью.

5. Consencus Patrum. Тот же Олег Давыденков далее пишет:

Естественно, могут возникнуть вопросы: как отличить подлинное учение отцов, имеющее авторитет, от частных мнений тех или иных отцов, в том числе и ошибочных ? Для этого существует специальное понятие: consensus patrum, согласие отцов. В святоотеческом учении принимается та его часть, по которой имеется единодушное мнение всех либо значительного большинства святых отцов ${ }^{4}$.

Здесь мы имеем дело с религиозной процедурой обоснования, позволяющей дополнительно усилить L-статус отцецерковности. Ее также можно представить как случай сравнительного обоснования, в котором эталоном выступает полное согласие отцов церкви (CР):

$$
\frac{\mathrm{CP}}{\mathrm{X} \mid \alpha \mathrm{CP}}
$$

Процедура измерения $\operatorname{deg}(\mathrm{CP}, \mathrm{X})=\alpha$ имеет здесь легко выразимую форму, прямо зависящую от доли реально согласных по некоторому вопросу X отцов Церкви. L-статус в этом случае мог бы быть назван отцеединством. 
6. Аналогия. В общем случае возможны, по-видимому, различные виды аналогий, используемые в обосновании религиозного опыта. Например, Йозеф Мария Бохеньский в работе Логика религии ${ }^{5}$ рассматривает аналогию как один из основных видов религиозного обоснования, опирающийся на тождество формальных свойств отношений. Я приведу здесь один из возможных примеров аналогии как случая сравнительного обоснования. Пусть $\mathrm{F}(\mathrm{a}, \mathrm{b})$ - отношение „b есть отец a”, $\mathrm{R}(\mathrm{a}, \mathrm{G})$ - некоторое отношение-переменная а и Бога $(G)$. В этом случае рассуждения о Боге как об отце для человека а используют следующее сравнительное обоснование:

$$
\frac{F(a, b)}{R(a, G) \mid \alpha F(a, b)}
$$

Здесь переменное отношение $\mathrm{R}(\mathrm{a}, \mathrm{G})$ человека а к Богу уподобляется на степень $\alpha$ конкретному отношению $\mathrm{F}(\mathrm{a}, \mathrm{b})$. Именно такое уподобление позволяет на степень $\alpha$ рассуждать о Боге как об отце, причем, по-видимому, степень $\alpha$ здесь не должна быть полной, выражая лишь аналогичность указанных отношений. В качестве эталона в этом случае выступает конкретное отношение $\mathrm{F}(\mathrm{a}, \mathrm{b})$, позволяющее в некоторой мере познать Бога, перенести на него отношение отцовства. Следовательно, L-статус здесь мог бы быть назван отцовской познаваемостью. Именно он в некоторой мере переносится с основания на репрезентат в этом примере.

\section{$\S 5$. Наука и религия как два типа организованной веры}

Как мы видим, в религиозном опыте христианства разного рода процедуры обоснования широко распространены. В этом смысле между наукой и религией наблюдается много сходного (хотя и на достаточно глубоком уровне обобщений), в согласии 
с аналогической моделью отношений религии и науки. Теперь более конкретно можно подойти к проблеме формулировки знания и веры как некоторых установок человеческого сознания. Я буду предполагать, что

1. Статическая вера выражает себя в отношении к основаниям в рамках той или иной процедуры обоснования. Вера - это и есть установка сознания на придание L-статуса основаниям - статуса обобщенной необходимости и безусловности.

2. Статическое знание, наоборот, выражает себя в отношении к репрезентатам в рамках той или иной процедуры обоснования, т.е. знание есть установка сознания на определение репрезентатов в М-статусе - статусе обобщенной условности и проблематичности.

В целом, любая процедура обоснования представляет из себя единство статической веры и знания, конкретно выражаемое в единстве оснований и репрезентатов, в двуплановости всякой процедуры обоснования. Кроме такого статического выражения веры и знания, можно дать их динамические определения.

$1^{*}$. Динамическая вера выразит себя в распространении L-статуса с оснований на репрезентаты, т.е. в проведении той или иной процедуры обоснования. С точки зрения репрезентатов, динамическая вера выразит себя как переход от M-статуса к L-статусу, как некоторое ML-преобразование репрезентатов.

$2^{\star}$. Динамическое знание, наоборот, будет выражать себя в распространении М-статуса, например, в критике тех оснований, которые ранее рассматривались как находящиеся в L-статусе. Для таких оснований динамическое знание предстанет как переход от L-статуса к M-статусу, т.е. как некоторое LM-преобразование оснований.

Такое деление требует введения представления также о некоторых процедурах антиобоснования, обратных процедурам обоснования. Для получения общего вида процедуры антиобоснования достаточно перевернуть структуру процедур обоснования, распространяя М-статус с репрезентатов на основания и выражая критическое отношение сознания к основаниям. 
Как наука, так и религия представляют собой примеры субъектных активностей, где постоянно взаимодействуют друг с другом процедуры обоснования и антиобоснования. Однако в религии несомненно в гораздо большей мере развиты процедуры обоснования, и динамическая вера здесь вполне господствует над динамическим знанием. В науке, по-видимому, мы имеем дело с более равновесным соотношением динамических веры и знания. Тем не менее, ранняя история христианства, борьба с ересями, уточнение и обновление догматики, необходимость согласования ряда онтологических положений вероучения с современными положениями науки также являют собою примеры использования процедур антиобоснования и динамического знания в христианстве (очевидно, что это положение вполне применимо и к другим видам религии).

В целом, как представляется, можно сделать вывод о единстве веры и знания - как в статическом, так и в динамическом аспекте - и в научном, и в религиозном опыте. В общем случае любой опыт представляет из себя сложную систему различных процедур обоснования-антиобоснования, находящуюся в более-менее относительном равновесии и способную к дальнейшему развитию.

Такие системы субъекто-бытия можно называть состояниями организованной веры, вкладывая в это название идею тех или иных форм и степеней взаимодействия веры и знания. Хотя Откровение-в-себе присутствует в составе религиозного опыта в форме первичного мистического факта, однако, Откровение-для-нас становится таковым как результат, по-видимому, всей полноты религиозных процедур обоснования. В этом смысле и наука и религия одинаково представляют из себя разновидности организованной веры, но конкретные типы этой организации конечно могут быть весьма различными. Как уже отмечалось, религиозный тип организованной веры тяготеет к преобладанию динамической веры над динамическим знанием. Кроме того, в религии можно говорить и о преобладании числа первичных оснований, которые сами уже не могут быть репрезентатами (на данном 
этапе развития) в других процедурах обоснования. В этом смысле можно говорить о явной тенденции к преобладанию и статической веры в религиозном опыте. Наука, по-видимому, наоборот стремится минимизировать число первичных оснований и максимизировать число возможных процедур антиобоснования, выражая этим преобладание статического и динамического знания над соответствующими видами веры. Тем не менее, по-видимому, законом функционирования и развития всякого субъектного бытия является взаимная дополнительность веры и знания во всех своих разновидностях, их сложное и тесное взаимодействие в единой целостности многополярной и всеединой Жизни. 